\section{Fine and gross motor ability in males with ADHD}

Thelma MI Pitcher BSc (Hons) Msc PhD);

Jan P Piek* BSc (Hons) PhD, Associate Professor; David A Hay MA (Hons) PhD, Professor of Psychology, School of Psychology, Curtin University of Technology, Perth, Nustralia.

*Correspondence to second author at School of Psychology, Curtin University of Technology, GPO Box U1987, Perth 6845, Australia.

E-mail: j.pick@curtin.edu.au

In the this study, both fine and gross motor ability of males with attention-deficit-hyperactivity disorder (ADHD) were compared with a group of control children. Three groups of males with the following ADHD subtypes: predominantly inattentive (ADHD-PI; $\boldsymbol{n}=50$ ), hyperactive/impulsive (ADHD-HI; $\boldsymbol{n}=\mathbf{1 6}$ ), or combined (ADHD-C; $\boldsymbol{n}=38$ ) were compared with 39 control males. Mean ages for the control group were 10 years 4 months (SD 1 year 4 months, range $\%$ years 8 months to 12 years 11 months); for the ADHD-PI group, 10 years (SD 1 year 2 months, range $y$ years 10 months to 13 years); for the ADHD-HI group, 9 years 11 months (SD 1 year 2 months), range 7 years 11 months to 12 years 6 months); and for the ADHD-C group 10 years 2 months (SD 1 year 4 months, range 8 to 13 years). The Australian Disruptive Behaviours Scale and Connors' Parent Rating Scale-Revised were used to assess ADHD symptomatology. Verbal IQ was estimated using two verbal subtests of the Wechsler Intelligence Scale for Children, and movement ability was assessed using the Movement Assessment Battery for Children (MABC) and the Purdue Pegboard test. Findings demonstrated that the children with ADHD had significantly poorer movement ability than control children. A high percentage of these children displayed movement difficulties consistent with developmental coordination disorder (DCD). In addition, the current study found that the type and degree of movement difficulty differed between subtypes. The Total Impairment score, as derived from the $\mathrm{MABC}$, was less severe for the ADHD-HI group than the other two ADHD groups, but more severe than for the control group. Males with ADHD-PI and ADHD-C had significantly poorer fine motor ability $(\boldsymbol{p}<0.001)$ than control males, whereas the ADHD-HI group did not differ significantly from any of the other groups. As children with ADHD only and the control group did not differ significantly on fine motor ability but were significantly better than children categorized with both ADHD and DCD, it was argued that poorer fine motor ability found in children with ADHD could not be attributed to deficits in attention and concentration, but rather to factors relating to their motor ability.

See page 535 for list of abbreviations.
Recent research has drawn attention to both the immediate and longer-term concomitant difficulties of children with motor problems such as developmental coordination disorder (DCD; Losse et al. 1991, Cantell et al. 1994, Piek et al. 2000, Skinner and Piek 2001). DCD is "characterized by impairment of motor performance sufficient to produce functional performance deficits not explicable by the child's | chronological] age or intellect, or by other diagnosable neurological or psychiatric disorders' (Polatajko ct al. 1995 p 5).

For over a century, children with motor problems have been associated with the behavioural clifficulties of inattention, hyperactivity, and impulsivity (Rutter 1982, Sandberg 1996) and there has been a general acceptance that approximately half of all children with attention-deficit-hyperactivity disorder (ADHD) may have motor difficulties (l lartsough and Lambert 1985, Barkley et al. 1990, Piek et al. 1999). However, motor dysfunction in these children remains underexamined (Doyle et al. 1995, Kadesjö and Gillberg 1998) and research investigating the relationship between ADHI) and ICD has been limited (Gillberg 1998).

The importance of such research is supported by the weight of evidence from the Swedish research groups which has detailed poorer outcomes for children with Deficits in Attention, Motor Control, and Perception (DAMP; Hellgren et al. 1994, Gillberg and Hellgren 1996, Rasmussen and Gillberg 2000). DAMP is viewed as an overarching concept that encompasses 'combinations of motor control and perceptual problems in conjunction with attentional problems encountered in children who do not show mental retardation or cerebral palsy' (Gillberg 1995 p 139). DAMP diagnostic criteria include the areas of attention, gross motor skills, fine motor skills, perceptual ability, and speech/anguage dysfunction (see (rillberg 1992 p 1323-24). Gillberg (1998) suggested that children with DAMP 'meet criteria of DSM-IV ADHI) (particularly the inattentive subtype) and "developmental coordination disorder" (p 108). Gillberg (1995) criticized studies within the attention-deficit syndrome paradigm for often overlooking 'concomitant neuropsychological and motor coordination problems' ( $p$ 40), even though there is general acceptance of the relationship between them. Indeed, some authors have suggested that the 'concept of AIJHI) may be clinically less relevant than that of DAMP' (Kadesjo and (iillberg 1998 p 803).

Bax and Whitmore (1987) argue that rather than focussing on specific learning disorders, we need to use a more general diagnosis such as neurodevelopmental disorder; which takes into account the involvement of both the nervous system and development in explaining the specific problems that a child may have, i.e. there is a general syndrome rather than a series of individual diagnoses.

Regardless of the approach taken to investigate psychopathology in children, there is a need to gain a better understanding of the relationship between $A D H D$ and motor coordination difficulties. The current study was designed to redress the relative absence of reliable and objectively assessed information pertaining to the motor abilities of children with subtypes of $\triangle D H D$ and to detail the association between ADHD symptomatology (i.e. inattentiveness and hyperactive-impulsive) and movement ability. 'Ihree subtypes of ADHD are identified by the DSM-IV (American Psychiatric Association 1994), namely: ADIHD predominantly inattentive type (ADHD-PI); ADHD predominantly hyperactive/impulsive (ADHD-HI); and ADHD, combined type (i.e. 
combined inattentive and hyperactive/impulsive symptoms; ADHD-C). 'The particular performance profiles for these subtypes remains relatively unknown in an atmosphere where both clinicians and researchers continue to question the precision of current diagnostic classifications and the variance in outcomes of cognitive abilities research. For example, Barkley (1997) suggested that ADHD-HI is a developmental precursor to AIHI)-C and is supported by Hart et al. (1995) who found that hyperactive/impulsive behaviour emerges early in the preschool years whereas the onset of inattentive symptomatology is several years later: Barkley (1997) has also suggested that ADHD-PI may not be an ADHD subtype but rather a separate disorder. Levy et al. (2001) on the other hand, have suggested that the three subtypes be considered as three genetically distinct disorders.

The primary aim of the current study was to investigate ADHD subtype-specific differences in motor ability. Previously we found that $30 \%$ of children with either ADHD-PI or ADHD$C$ subtypes performed at a level consistent with DCD, and another $30 \%$ of each group were in need of monitoring their motor performance (i.e. classified 'at risk' of DCD) and its life consequences (Piek et al. 1999). The children with ADHD in this carlice study (i.c. ADHD) PI and ADHI)-C) had significantly poorer general movement ability than did the comparison group. We did not, however, include a group of children with ADHD-HI in that study and the current study was designed to investigate motor ability profiles in all three ADHD subtypes.

In the earlier study (Piek et al. 1999) we also found that males with ADHD-PI had significant fine motor difficulties, whereas the primary difficulties of males with ADHD-C were of a gross motor origin. This was further investigated in the current study, firstly through the analysis of the balance, ball skills, and manual dexterity subcomponents of the Movement Assessment Battery for Children (MABC; Henderson and Sugden 1992), and secondly via the analysis of data derived from an additional test of fine motor skill: the Purdue Pegboard test (Tiffin and Asher 1948, Tiffin 1968).

$A$ further aim was to examine the impact on fine motor ability of having both ADHD and DCD. It has been suggested that poorer fine motor ability in children with ADHD is linked to the fact that fine motor skills 'make greater demands for sustained attention and effortful activity' (e.g. Doyle et al. 1995 p 237). It would, therefore, be expected that even if children with $A D H D$-PI or ADHD-C are not diagnosed with DCD, their fine motor movements would be poorer than control children as a result of their inattentive symptomatology. This was investigated using the Purdue Peg Board test.

Only males were included in the current study. Justification for a sex specific investigation is found on many levels. First, subtype prevalence estimates indicate that although males continue to constitute the greater percentage of children within each ADHD subtype, females have a higher proportional representation within the ADHD-PI subtype (Lahey et al. 1994). Second, sex differences have been reported for motor performance (Henderson and Hall 1982) but have not been adequately addressed in the literature. Reports vary as to whether males experience more severe motor coordination problems than females (Henderson and Hall 1982, Hoare and Larkin 1991). Culturally influenced performance expectations (Hoare and Larkin 1991), increased visibility of physical performance (Smyth 1992), sex-related interaction styles, and social environment have been found to influence motor skill acquisition (Garcia 1994) and the identification of motor problems. Sex differences may also be influenced by stimulus configuration (Laszlo et al. 1980), visuo-spatial cue processing (Livesey and Intili 1996), and vividness of movement imagery (Issac and Marks 1994). In combination these issues led to the decision to reduce potential confounds by focussing on males for the current study.

If motor difficulties are confirmed to be problematic, then given that previous research has shown negative psychosocial and academic outcomes for children with DCD and DAMP, it may be appropriate to include motor assessment and intervention in 'best practice' regimes for children with ADHD.

\section{Method}

PARTICIPANTS

One hundred and fifty seven males (mean age 10 years 1 month, SD 1 year 4 months; age range 7 years 8 months to 12 years 11 months) participated in the study. Participants were recruited from 35 mainstream primary schools within the Perth metropolitan area in Western Australia, and were not involved in the earlier study (i.e. Piek et al. 1999).

MEASURES

Australian Disruptive Behaviours Scale

The shortened form of the Australian Disruptive Behaviours Scale (ADBS; Levy and Hay 2001) is a parent-report questionnaire that lists items reflective of DSM-IV (American Psychiatric Association 1994) criteria for ADHD (i.e. 9 inattentive symptoms and 9 hypcractive/impulsive symptoms). A standard set of instructions directs the rater to indicate the applicability of each item for their child, either now or within the last six months. Items are rated on a scale of 0 (not at all) to 3 (very much/very often). Ratings of 0 or 1 are interpreted as symptom absent and ratings of 2 or 3 are interpreted as symptom present. This method of establishing symptom presence is consistent with the procedures adopted in other studies (Pelham et al. 1992, Lahey ct al. 1998). 'This procedure has produced similar estimates of the prevalence of the three subtypes and of the latent structure, when comparing Australian and Missouri samples (Rasmussen et al. 2002). The ADBS ratings of behaviour have been found to be a conservative indicator of ADHD symptom presence (Levy et al. 1996) in that more symptoms were reported when assessed by interview compared with the ADBS rating. Studies using DSM-III-R (American Psychiatric Association 1987) ADHD criteria have reported kappa coefficients of 0.561 to 0.648 and an alpha coefficient of 0.86 (Levy et al. 1997). The ADBS has been found to be a reliable measure of the presence of inattentive symptoms $(r=0.93)$ and hyperactive/impulsive symptoms ( $r=0.95$; Levy et al. 2001). Dimensional scores may be calculated by summing the participant's relevant responses to provide an overall rating of inattention and hyperactivity/impulsivity.

\section{Conners Parent Rating Scale-Revised}

The long form of the Conners' Parent Rating Scale-Revised (CPRS-R:L; Conners 1997) is for use with children aged from 3 to 17 years, takes 15 to 20 minutes to complete and was used in order to substantiate the ADHD groupings. The 80 -item measure produces a seven factor outcome (i.e. cognitive problems, oppositional behaviour, hyperactivity-impulsivity, anxiety and shyness, perfectionism, social problems, and psychosomatic 
behaviour) with internal reliability coefficients ranging from 0.74 to 0.94 for males (Conners et al. 1998).

\section{Wechsler Intelligence Scale for Children-III}

A short form of the Wechsler Intelligence Scale for Children-III (Wechsler 1992; Vocabulary and Similarities subtests) was used to derive a prorated verbal intelligence (VIQ) score in order to ensure comparative abilities between groups. These two subtests were chosen because as a dyad they have high reliability (Vocabulary $r=0.87$; Similarities $r=0.81$ ) and validity coefficients (Sattler 1992, Wechsler 1992) and have been used in other studies of motor performance (Henderson and Hall 1982, Lord and Hulme 1987, Piek and Coleman-Carman 1995, Piek et al. 1999). The subtests are the furst (Vocabulary) and third (Similarities) best measures of general cognitive ability (Sattler 1992).

\section{Movement Assessment Battery for Children}

The Movement Assessment Battery for Children (MABC; Henderson and Sugden, 1992) is a standardized, two part, structured, motor ability assessment consisting of a parent/teacher report checklist measuring everyday movement ability and an individually administered performance test. 'The standardized performance test was used in the current study. It consists of four age bands (4-6,7-8,9-10, and 11-12 years) that incorporate the performance of eight different tasks rated on a 0 to 5 scale. The tasks are grouped in three performance groupings of: Manual Dexterity (a measure of fine motor ability, 3 tasks), Ball Skills (a measure of both fine and gross motor ability, 2 tasks), and Static and Dynamic Balance (a measure of gross motor ability, 3 tasks). Four scores may be derived: a Total Impairment score and a score profiling performance within each grouping of tasks. Higher scores indicate greater motor impairment. Children with total Impairment scores in the top 5 th centile are considered to have performed at a level 'indicative of a definite motor problem' that requires intervention (Henderson and Sugden, 1992 p 108). Children with scores between the 85 th and up to the 95 th centile demonstrate a borderline' degree of difficulty (Henderson and Sugden 1992).

The MABC is a revised version of the lest of Motor Impairment (ГOMI; Stott et al. 1984). 'TOMI test-retest and interrater reliability have been assessed to be equal to or greater than 0.75 and 0.70 respectively in a study involving 360 randomly selected children of all ages. The percentages of agreement between a test-retest study for the MABC revealed a range between 73 to $97 \%$ concordance in the Total Impairment score and similarly in each of the three performance groupings (Fenderson and Sugden 1992). A recent study has reported the MABC to correlate significantly $(r=0.62)$ with a German test of coordination in children (Körperkoordinations Test für Kinder; Kiphard and Schilling 1974 cited in Smits-Engelsman et al. 1998).

\section{Purdue Pegboard}

The Purdue Pegboard test (Tiffin and Asher 1948, Tiffin 1968) was designed to measure finger and hand dexterity. The test was originally designed as a personnel selection test but has more recently been used as a neuropsychological measure (Sattler 1992). The apparatus consists of a board with two parallel rows of 25 holes each. Pegs, collars, and washers are located in four cups at the top of the board (Figure 1).

The first three subtests require the participant to place as many pins as possible in the 25 holes within 30 seconds, first with the preferred hand, then the non-preferred hand, and finally with both hands. In the fourth subtest the participant must use both hands alternatively (beginning with the preferred hand) to build as many 'assemblies' (i.e. a pin, a washer, a collar, and another washer) as possible within one-minute. Scores are derived for each part of the test. The majority of reliability and validity studies have been performed with adults in various occupations recuiring manual dexterity skills (liffin 1968). Reported reliabilities range between 0.60 to 0.76 for $\sin$ gle trial scores and 0.82 to 0.91 for three trial scores ('Tiffin 1968). The Purdue Pegboard test has been used with children with learning disabilities* (Costa et. al. 1963 cited in Tiffin 1968) and has been found to distinguish reliably the performance of children with minimal brain dysfunction from control children across a 5 to 15 years 11 months age range (Cardnex and Broman 1979). The apparatus has also been used within studies comparing the timed motor performance of children with DCD with control children (e.g. Lyytinen and Ahonen 1989, Cantell 1998).

\section{PROCIDLRE}

Fthical guidelines of the National Health and Medical Research Council of Australia were followed with particular emphasis on those pertaining to the testing of children. The Head teachers of 35 Perth primary schools were sent recruitment literature containing a participant information sheet, a parent/guardian consent form, and a reply-paid envelope for distribution to all males in Grades 3 to 7 . Consenting parents/guardians were forwarded a letter and questionnairc (i.e. the ADBS and a descriptive questionnaire). Three hundred and thirty one questionnaires were returned: a return rate of $9.2 \%$. The initial letter also requested that non-consenting parents/guardians provide feedback about their reasons for

\section{*US usage: mental retardation}

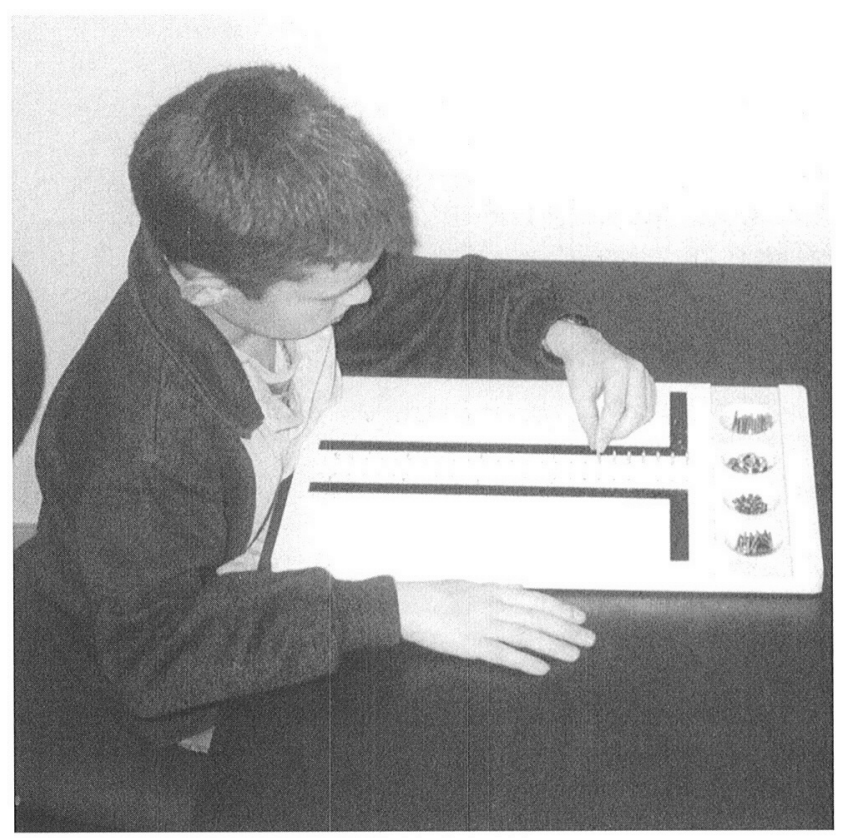

Figure 1: Purdue Pegboard test: a measure of finger and band dexterity (Tiffin 1968). 
non-participation. Consistent with previous research (c.g. August et al. 1992), the main reasons for non-participation were the time, travel or work/school/family commitments, or a lack of child consent.

The questionnaire was used to screen participants and allocate group membership as described later. The parent/guardian of any child who completed the entire testing process was also asked to complete the relevant CPRS-R:I. Parents were notified upon completion of the study if there were any indications that their child may have motor coordination difficulties, the presence of a high degree of ADHI symptomatology, or any concerning elevation in associated clinical problems as identified by the CPRS-R:L.

'The majority of testing sessions were conducted at the participant's school and took approximately one hour to complete. A small percentage of children were tested at home or at the University. Each session commenced with administration of the WISC-III Vocabulary and Similarities subtests. The participants then completed the PPB and MABC tests. The process of administration of these tests occurred in a counterbalanced format to control for both practice effects and fatigue.

In order to minimize the effects of medication on test outcomes, all males currently taking medication were asked to abstain from all doses on their scheduled day of testing. In addition, every effort was made to coordinate testing with any usually occurring temporary withdrawals (e.g. school holidays). Adopting this procedure meant that all medicated participants had, at minimum, a period of abstinence of approximately 15 hours before being tested.

\section{Attention lo task}

As an objective estimate of group levels of attention to task, a random sampling technique was used to select up to 10 participants from each group for behavioural analysis. Each selected participant was videotaped for three 5 minute periods beginning at the 10th, 30th, and 50th minute of the testing process. A psychologist and a mental health practitioner then rated the video scgments using the child observation component of the Observing Pupils and 'leachers In Classrooms system (OPTIC; Iloughton et al. 1990). 'The OP'IC system has proven to be a reliable and valid method of behavioural assessment and enables the derivation of a 'percentage of on-task behaviour' score for each observed participant (Merrett and Wheldall 1986). 'The interrater reliability of the 'on-task' ratings in the current study was significant at $r=0.98, p<0.001$ (off-task ratings, $r=0.95, p<0.001$ ). There was no significant difference in the mean level of on-task $(F[3,52]=2.24, p>0.05)$, nor off-task $(F[3,52]=2.24, p>0.05)$ behaviour. The per group mean ontask percentages for each group were: control group, $98 \%$; ADHD-PI 98\%; ADHD-HI 95\%; and ADHD-C 92\%.

DATA ANALYSIS

Data were analyzed using two distinct approaches to participant groupings.

\section{SUB'YYPI COMPARISONS}

Each participant's primary group membership classification was based on the parent/guardian response to the short form of the ADBS. Males classified in the ADHD-PI group $(n=50)$ were reported to have at least six of nine inattentive symptoms but less than 6 hyperactive/impulsive symptoms. Forty percent of the AISHD-PI group had been previously diagnosed. Males in the ADHD-HI group ( $n=16$ ) had at least six of nine hyperactive/impulsive symptoms but fewer than six inattentive symptoms (37.5\% had received a previous diagnosis). Males in the ADHD-C group $(n=38)$ had at least 6 of 9 symptoms within both the inattentive category and the hyperactive/impulsive category (66\% had received a previous diagnosis). A smaller number of males were classified with ADHD-HI than either ADHD-PI or ADHI)-C. 'This was an anticipated outcome considering that children with ADHD-HI are generally more prevalent in a younger age range (Lahey et al. 1994) than that considered ideal for the current study. Six males with a previous ADHD diagnosis failed to meet the ADHD rating scale inclusion criteria. Another eight males had high presence of ADHID symptoms (e.g. five inattentive symptoms and five hyperactive/impulsive symptoms) but failed to moet the minimum diagnostic criteria (i.e. six of nine symptoms).

Participants allocated to the control group $(n=39)$ were required to have minimal ADHD symptomatology ( $<3$ ADHI) symptoms), minimal pre- and postbirth complications, and no serious previous or existing developmental and/or intellectual difficulties.

Parents/guardians also completed the CPRS-R:I, with the results providing support for the ADHD groupings. Significant group differences were found for oppositional behaviour $(F[3,136]=35.41, p<0.001)$, hyperactivity $(F[3,136]=59.41$, $p<0.001)$, and restless/impulsive index $(F[3,136]=85.72$, $p<0.001$ ). Post-hoc analysis revealed that all four groups differed from each other with respect to these dependent variables with the exception of the ADHD-( and ADHI)-HI groups. As expected, these two groups had the highest scores, as disruptive behaviours have a strong link with hyperactive/impulsive symptomatology. Significant group differences were found for cognitive problems or inattention difficulties, $(F[3$, $136]=79.01, p<0.001)$. Post-hoc analysis indicated that all groups differed from each other with the exception of ADHDPI and ADHD-C groups. Again, this is to be expected as these are the two groups with inattentive symptomatology. The Conners' ADHD Index, which is a measure of the level of 'risk' for ADHD, significantly differentiated the groups, $(F[3,136]=90.29, p<0.001$. Post-hoc analysis indicated that the ADHD-C group was more at risk for ADHD than any of the other groups. All groups significantly differed from each other on this measure with the exception of the ADHD-PI and ADHD -HI groups.

'abble I displays the group means, standard deviations (SDs), and range for the number of ADHD symptoms, age, and prorated WISC-III VIQ based on the scores from the Vocabulary and Similarities subtests (Wechsler 1992). Age, $(F[3,139]=0.574$, $p>0.05)$, and VIQ, $F(3,139)=1.97, p>0.05$, were analyzed separately and neither variable was found to differentiate the groups significantly. Family related information demonstrated that measures of socioeconomic status did not significantly differentiate the groups $(F[9,417]=1.33, p>0.05)$.

Dependent variables used in comparing ADHD subtypes were divided into three categories: (1) MABC' Total Impairment score; (2) MABC sub-components, and (3) PPB tests.

\section{MABC Total Impairment score}

Statistical significance was assessed using a univariate analysis of variance (ANOVA) in order to determine whether groups differed on the MABC Total Impairment score. There were violations of the assumption of variance. This can be 
partially explained by the fact that the MABC (Henderson and Sugden 1992) was designed for diagnostic purposes and, as a result, the range for good to exceptional performance may be somewhat restricted. logarithmic transformations were performed in an attempt to reduce variability and general skew of the data. However, this had little effect, and for clarity of interpretation the non-transformed data are presented. As a result, violations of the assumptions for homogeneity of variance necessitated an adjustment to the per comparison alpha level to compensate for any effects on Type I error (i.e. $\alpha=0.025$ ). Planned comparisons, evaluated at a modified Bonferroni corrected alpha level of 0.0375 (i.e. $3 \times 0.05 / 4$ ) and corrected for assumptions of unequal variance, were conducted to compare groups.

Participants were also classified into one of three 'ability' categories according to their MABC Total Impairment score: (1) acceptable performance, Total Impairment score $<85$ th centile; (2) borderline performance, Total Impairment score $\geq 85$ th and $<95$ th centile; and (3) 'DCD', 'Total Impairment score $\geq 95$ th centile.

A unidirectional $\chi^{2}$ test of relatedness was used to examine the association between experimental group and MABC performance category.

\section{MABC subcomponents}

These subcomponents consist of manual dexterity, ball skills, and balance. MANOVA, followed by three separate univariate ANOVAs, were used to relate group membership to each of the subcomponents of the MABC. Multivariate and univariate homogeneity of variance assumptions for ball skills and static and dynamic balance were violated. Again, as logarithmic transformations did not correct this, an adjustment was made to the per comparison alpha level $(\alpha=0.025)$ for the respective analyses. Planned comparisons evaluated group performance for significant univariate effects at a modified Bonferroni corrected alpha level of 0.025 .

\section{PPB Tests}

The dependent variables consisted of: (1) PPB Composite score, i.e the combined score for dominant hand, non-dominant hand, and both hands together as a measure of fine motor ability; (2) PPB Dominant Hand score, a measure of dominant hand fine motor proficiency; (3) PPB Non-dominant Hand score, a measure of non dominant hand fine motor proficiency; (4) PPB Both Hands Together score, a measure of fine motor proficiency in a bimanual task; and (5) PPB Assembly Task score, a measure of fine motor proficiency and planning ability in a complex bimanual task.

Firstly, a four-group ANOVA was used to assess the relationship of group membership to the PPB composite score. The significant outcome was followed by planned comparisons to evaluate the predicted group difference on PPB composite score. Statistical significance was assessed at a modified Bonferroni corrected alpha level of 0.03 . 'The predicted outcomes for the remaining PPB variables (i.e. PPB Dominant, Non-dominant, Both Yands, and Assembly scores) were analyzed by means of a $4 \times 4$ MANOVA due to their conceptual relatedness.

ADHD/DCD, ADHD, ANI) COMPARISON GROUP

In this grouping, membership involved the categorization of the ADHD and comparison participants into groups based on the presence or absence of a DCD classification. 'The performance criteria for DCD classification was based on the participant's MABC Total Impairment score (i.c. 285 th centile). A DCD classification suggested a range of movement ability that was either indicative of a need for ongoing monitoring of the impact of difficulties in the participant's daily life, or when toward the higher end of the centile range, indicated a need for intervention.

The first group included all males who met the criteria for ADHD categorization and a 'DCD' classification (i.e. ADHD/DCD). The ADHD/DCD group comprised 55 participants. A second group consisted of all participants with an

Table I: Means, SDs, and range for age, VIQ, number of DSIV-IV ADHD symptoms, and ADHD symptomatology continuum score for groups

\begin{tabular}{|c|c|c|c|c|c|c|}
\hline \multirow[t]{2}{*}{ Group } & \multirow[t]{2}{*}{ Age, $y: m$} & \multirow[t]{2}{*}{$V I Q$} & \multicolumn{2}{|c|}{ DSM-IV symptoms } & \multicolumn{2}{|c|}{ Continuum symptoms } \\
\hline & & & $A D H D-P I$ & $A D H D-H I$ & $A D H D-P I a$ & $A D H D-H I^{b}$ \\
\hline \multicolumn{7}{|c|}{ Comparison $(n=39)$} \\
\hline Mean & $10: 4$ & 108.54 & 0.15 & 0.13 & 3.85 & 2.26 \\
\hline SD & $1: 4$ & 18.29 & 0.59 & 0.41 & 2.57 & 2.19 \\
\hline Range & $7: 8-12: 11$ & $70-141$ & $0-3$ & $0-2$ & $0-11$ & $0-8$ \\
\hline \multicolumn{7}{|c|}{ ADHD-PI $(n=50)$} \\
\hline Mean & 10:0 & 101.68 & 7.50 & 2.12 & 19.62 & 8.70 \\
\hline SD & $1: 2$ & 19.72 & 1.23 & 1.85 & 3.72 & 4.96 \\
\hline Range & $7: 10-13: 0$ & $59-137$ & $6-9$ & $0-5$ & $15-27$ & $0-18$ \\
\hline \multicolumn{7}{|c|}{ ADHD-HI $(n=16)$} \\
\hline Mean & $9: 11$ & 99.50 & 2.87 & 6.81 & 11.06 & 18.44 \\
\hline SD & $1: 2$ & 19.69 & 1.50 & 1.05 & 3.49 & 3.03 \\
\hline Range & $7: 11-12: 6$ & $54-133$ & $1-5$ & $6-9$ & $5-17$ & $14-24$ \\
\hline \multicolumn{7}{|c|}{ ADHD-C $(n=38)$} \\
\hline Mean & $10: 2$ & 98.63 & 8.16 & 7.82 & 22.03 & 21.03 \\
\hline SD & $1: 4$ & 18.98 & 1.08 & 1.04 & 3.45 & 3.62 \\
\hline Range & $8: 0-13: 0$ & $65-133$ & $6-9$ & $6-9$ & $14-27$ & $15-27$ \\
\hline
\end{tabular}

asum of nine symptom responses on 0 to 3 rating scale of Australian Disruptive Behaviour Scale (Levy and Hay 2001$)$ out of a possible 27. VIQ, verbal intelligence quotient; ADHD-PI, predominantly inattentive ADHD; ADHD-HI, hyperactive/impulsive ADHD. 
ADHII) categorization but no 'DCD' classification (i.e. MABC 'Total Impairment score $<85$ th centile). 'These males werc diagnosed with 'pure' DSM-IV ADHD with one of the three subtypes (i.e. ADHD; $n=49$ ). ADHD subtypes were not separated into different groups as this would have resulted in small group numbers. The comparison group comprised all participants without either ADHD or DCI) ( $n=31)$. A 'pure' DCD group was not formed due to low numbers (i.e. only eight participants from the original comparison group met DCD criteria).

Means, SISs, and range for age, prorated VIQ, and categorical and continuum based ADHD symptom scores are displayed in 'lable II. As the groups were subject to reformulation, the differences in age and VIQ were evaluated. Age differences between groups were found to be statistically nonsignificant $(F[2,132]<1)$. However, a statistically significant difference was found for VIQ, $(F[2,132]=4.96, p<0.025)$. Post-hoc analysis revealed that the ADHD/DCD group had significantly lower vre than the comparison group $(p<0.05$.) Both the $\mathrm{ADHD} / \mathrm{OCD}$ and $\mathrm{ADHI}$ groups had significantly more inattentive and hyperactive/impulsive symptomatology than did the comparison group $(p<0.05)$ but they did not differ between themselves $(p>0.05)$.

The dependent variables examined using this group allocation are described above and consist of: PPB Composite score; PPB Dominant Hand score; PPB Non-dominant Hand score; PPB Both Hands Together score; and PPB Assembly lask score. Given that the ADHD/DCD group had a significantly lower VIQ than the comparison group and that VIQ significantly correlated with each of the PPB variables, VIQ was used as a covariate. A three-group ANCOVA was used to assess the relationship of group membership to the PPB Composite score. The predicted outcomes for the remaining PPB variables (i.e. PPB Dominant, Non-dominant, Both Hands, and Assembly scores) were analyzed by means of a $3 \times 4$ MANCOVA. The significant MANCOVA was followed by four separate univariate ANCOVAs. A Bonferroni corrected alpha level was used where appropriate, in order to maintain a family-wise alpha of 0.05 . Significant findings were followed up using planned contrasts across the marginal means for the group factor at the modified Bonferroni corrected alpha level of 0.03 .

\section{Results}

SUB'TYPI: COMPARISONS

\section{MABC Total Impaiment score}

Table III displays the cell means, SDs, and range for group 'lotal Impairment scores on the MABC. 'I'he obtained $F$ ratio from the univariate ANOVA was statistically significant $(F[3,139]=5.89$, $p<0.025$ ), indicating that the groups significantly varied according to their overall performance on the $\mathrm{MABC}$. Planned comparisons indicated, first, that the three ADHD groups had a higher mean impairment score than the control group, $(l[95.79]=-4.46, p<0.001)$. Also, mean performance of the ADHD-PI and ADHD-C groups was significantly worse than that of the comparison group, $(t[113.93]=-4.94, p<0.001)$. However, the mean ability of the ADHD-PI and ADHD-C groups was not significantly different to that of the ADHD. HI group $(t[25.70]=-1.48, p>0.0375)$. Also, the ADHD-HI group's mean Total Impairment score did not significantly differ from that of the comparison group $(t[21.71]=-1.74$, $p>0.0375$ ).

'lable IV displays the percentage of participants from each group found to have either acceptable, borderline, or 'DCD' movement ability. 'The $\chi^{2}$ test was statistically significant, $\chi^{2}(6$, $\mathrm{N}=43)=14.55, p<0.025$, indicating that group membership will significantly influence performance category membership.

\section{MABC subcomponents}

Iable $V$ displays the cell means, SDs, and range of performance for each group on the variables manual dexterity, ball skills, and static and dynamic balance. The $4 \times 3$ MANOVA was significant $(F[9,417]=2.36, p<0.025)$, indicating that the linear combination of the subcomponents of the MABC significantly differentiated group membership.

\section{Manual Dexterity}

There was a significant group effect for manual dexterity $(F[3,139]=3.45, p<0.025)$. Planned comparisons showed that the fine motor ability (i.e. as measured by the MABC Manual Dexterity tasks) of both the ADHD-PI group $(\ell[139]=-3.05$, $p<0.025)$, and the ADHD-C group $(t[139]=-2.47, p<0.025)$, was significantly poorer than that of the comparison group. However, the mean Manual Dexterity score of the ADHI-HI

Table II: IVeans, SDs, range for age, VIQ, number of DSIVI-IV ADHD symptoms and ADHD symptomatology continuum score for $\mathrm{ADHD} / \mathrm{DCD}, \mathrm{ADHD}$, and comparison groups

\begin{tabular}{|c|c|c|c|c|c|c|}
\hline \multirow[t]{2}{*}{ Group } & \multirow[t]{2}{*}{ Age, $y: m$} & \multirow[t]{2}{*}{$V I Q$} & \multicolumn{2}{|c|}{ DSM-IV Symptoms } & \multicolumn{2}{|c|}{ Continuum Symptoms } \\
\hline & & & $A D H D-P I$ & $A D H D-H I$ & $A D H D-P I a$ & $A D H D-H I^{b}$ \\
\hline \multicolumn{7}{|c|}{ Comparison $(n=31)$} \\
\hline Mean & $10: 2$ & 111.10 & 0.13 & 0.13 & 3.61 & 2.29 \\
\hline SD & $1: 5$ & 17.98 & 0.56 & 0.43 & 2.47 & 2.27 \\
\hline Range & $7: 8-12: 11$ & $70-141$ & $0-3$ & $0-2$ & $0-11$ & $0-8$ \\
\hline \multicolumn{7}{|c|}{$\mathrm{ADHD}(n=49)$} \\
\hline Mean & $9: 11$ & 103.06 & 7.00 & 5.18 & 19.63 & 15.55 \\
\hline $\mathrm{SD}$ & $1: 4$ & 17.89 & 2.35 & 3.25 & 5.27 & 7.69 \\
\hline Range & $7: 10-13$ & $59-133$ & $1-9$ & $0-9$ & $6-27$ & $2-27$ \\
\hline \multicolumn{7}{|c|}{$\mathrm{ADHD} / \mathrm{DCD}(n=55)$} \\
\hline Mean & $10: 2$ & 97.71 & 7.05 & 4.69 & 18.78 & 13.95 \\
\hline $\mathrm{SD}$ & $1: 2$ & 20.32 & 2.03 & 2.97 & 4.95 & 6.76 \\
\hline Range & $7: 10-12: 6$ & $54-137$ & $1-9$ & $0-9$ & $5-27$ & $0-26$ \\
\hline
\end{tabular}

asum of nine symptom responses on 0 to 3 rating scale of Australian Disruptive Behaviour Scale (Levy and Hay 2001 ) out of a possible 27. VIQ, verbal intelligence quotient; ADHD-PI, predominantly inattentive ADHD; ADHD-HI, hyperactive/impulsive ADHD. 
group was not significantly worse than the score of the comparison group $(t[139]=-1.46, p>0.025)$. Furthermore, planned comparisons indicated that none of ADHD groups differed significantly from each other in terms of average fine motor ability.

\section{Ball Skill}

There was a significant group difference for ball skills $(F[3,139]=5.49, p<0.005)$. Planned comparisons indicated that the average ball skills ability of both the ADHD-PI group $(t[71.48]=-4.17, p<0.025)$ and the ADHD-C group $(t[53.92]=-3.03, p<0.025)$, was significantly poorer than that of the comparison group. Although the ball skills ability of the ADHD-HI group did not significantly differ from that of the comparison group $(t[25.09]=-1.2, p>0.025)$, it was significantly better than that of the ADHD-PI group $(t[51.02]=-2.55$, $p<0.025)$. The ADHD-C group's ball skills score did not significantly vary from either the ADHD-HI $(t[47.37]=-1.65$, $p>0.025)$ or the ADHD-PI $(t[83.37]=0.82, p>0.0250)$ group scores.

\section{Static and dynamic balance}

Univariate $A N O V A$ revealed no significant group effect for static and dynamic balance $(F[3,139]=2.49, p>0.025)$. However, statistical power was low $(0.61)$, and effect size was small (partial $\mu^{2}=0.051$ ).

\section{Purdue Pegboard}

Table VI displays the means, SDs, and range of performance for each group on the dependent variables that constitute the sub-components of the PPB.

The obtained $F$ ratio for the PPB Composite score was statistically significant $(F[3,139]=3.04, p<005)$, indicating that the groups significantly differed with respect to their combined right hand, left hand, and both hands performance on the PPB. Statistically significant planned comparisons found that the ADHD-PI group's mean PPB Composite score was significantly poorer than the comparison group's mean score $(t[139]=2.98, p<0.03)$. All other group comparisons were statistically nonsignificant with small effect sizes.

A $4 \times 4$ MANOVA was used to evaluate group differences on the remaining PPB variables (i.e. PPB Dominant, Non-dominant, Both Hands and Assembly scores). Against prediction, the multivariate outcome indicated that the linear combination of the PPB variables did not significantly differentiate the groups $(F[12,414]=1.22, p>0.05)$

ADHD/DCI, ADHD, ANI COMPARISON GROUP Table VII displays the means, SDs, and range of performance for each group on the PPB dependent variables.

The ANCOVA for the PPB Composite score was statistically significant $(F[2,131]=12.18, p<0.01)$. Planned contrasts confirmed the significantly poorer performance of the ADHD/DCD group as compared with the comparison group or the ADHD group on the PPB Composite measure $(p<0.03)$. The contrast between the comparison group and $\mathrm{ADHD}$ group was found to be statistically nonsignificant $(p>0.03)$.

For the remaining PPB variables (i.e. PPB Dominant, Nondominant, Both Hands and Assembly scores) the obtained $F$ ratio for the MANCOVA indicated that the linear combination of the PPB variables significantly differentiated the groups $(F[8,258]=2.72, p<0.01)$. Univariate analyses demonstrated that the obtained $F$ ratios for all $P P B$ variables were statistically significant $(p<0.01$; see Table VIII).

The planned contrasts confirmed that the ADHD/DCD group was significantly poorer in performance on every PPB variable as contrasted with both the comparison group and the ADHD group $(p<0.03)$. The comparison group and the ADHD group did not significantly differ from each other on any of the PPB variables $(p>0.03)$.

\section{Discussion}

Two main aims were addressed in this paper. First, ADHD) subtype-specific motor performance profiles were established

Table III: Means, SDs, and range for groups on Total Impairment score of Movement Assessment Battery for Children (Henderson and Sugden 1992)

\begin{tabular}{lrrrr}
\hline $\begin{array}{r}\text { Comparison } \\
(n=39)\end{array}$ & $\begin{array}{r}A D H D-P I \\
(n=50)\end{array}$ & $\begin{array}{r}A D H D-H I \\
(n=16)\end{array}$ & $\begin{array}{r}A D H D-C \\
(n=38)\end{array}$ \\
\hline $\begin{array}{l}\text { Total Impairment score } \\
\quad\end{array}$ & 6.47 & 12.09 & 9.25 & 11.32 \\
Mean & 4.17 & 7.51 & 5.78 & 7.70 \\
SD & $0-15$ & $0.5-40$ & $1-18$ & $0-33$ \\
Range & & & & \\
\hline
\end{tabular}

${ }^{\mathrm{a}}$ Lower the score the better the performance.

Table IV: Percentage of participants in Movement Assessment Battery for Children (Henderson and Sugden 1992) motor ability performance categories by group

\begin{tabular}{lrrrr}
\hline Performance & $\begin{array}{r}\text { Comparison } \\
(n=39)\end{array}$ & $\begin{array}{r}\text { ADHD-PI } \\
(n=50)\end{array}$ & $\begin{array}{r}\text { ADHD-HI } \\
(n=16)\end{array}$ & $\begin{array}{r}\text { ADHD-C } \\
(n=38)\end{array}$ \\
\hline Acceptable & 79.5 & 42.0 & 50.0 & 52.6 \\
Borderline & 10.3 & 16.0 & 18.8 & 18.4 \\
'DCD' & 10.3 & 42.0 & 31.3 & 28.9 \\
Total with problem & 20.5 & 58.0 & 49.1 & 47.3 \\
performance & & & &
\end{tabular}

${ }^{\mathrm{a}} \mathrm{S}$ cores in upper 15 th centile.

Table V: Means, SDs, and range for groups on manual dexterity, ball skills, and static and dynamic balance

\begin{tabular}{lrrrr}
\hline Skill & $\begin{array}{r}\text { Comparison } \\
(n=39)\end{array}$ & $\begin{array}{r}\text { ADHD-PI } \\
(n=50)\end{array}$ & $\begin{array}{r}\text { ADHD-HI } \\
(n=16)\end{array}$ & $\begin{array}{r}\text { ADHD-C } \\
(n=38)\end{array}$ \\
\hline $\begin{array}{l}\text { Manual dexterity }{ }^{\mathrm{a}} \\
\text { Mean }\end{array}$ & 4.01 & 6.34 & 5.56 & 6.03 \\
SD & 0.49 & 0.56 & 0.81 & 0.59 \\
Range & $0-12$ & $0-15$ & $1-11.5$ & $0-13$ \\
Ball skills ${ }^{\text {a }}$ & & & & \\
Mean & 0.54 & 2.28 & 1.00 & 1.84 \\
SD & 0.19 & 0.37 & 0.34 & 0.39 \\
Range & $0-4$ & $0-10$ & $0-4.5$ & $0-9$ \\
Static and dynamic balance & & & & \\
Mean & 1.92 & 3.50 & 2.81 & 3.39 \\
SD & 0.29 & 0.46 & 0.60 & 0.57 \\
Range & $0-8$ & $0-15$ & $0-7$ & $0-15$ \\
\hline
\end{tabular}

${ }^{\mathrm{a}}$ Lower the score the better the performance. 
following the assessment of motor performance on the MABC (Henderson and Sugden 1992) and PPB (Tiffin and Asher 1948, Tiffin 1968). Also of interest was the outcome on the PPB for participants with both ADHD and DCD compared with those diagnosed with only ADHD.

The first analysis involved a comparison between the three ADHD subtypes and a control group regarding the mean MABC Total Impairment scores. The ADHD-PI and ADHD-C groups, and not the ADHD-HI group, differed significantly from the comparison group in their lotal Impairment score. This suggests that the subtypes that include inattentive symptomatology may be the ones most affected by poor motor performance. That is, if a purcly categorical approach to group identification is used then this outcome may lead one to assume that the ADHI)-HI group (who by categorical definition do not have significant inattentive difficulties) was not significantly more impaired than the comparison group due to the relative absence of inattentive symptomatology. However, the significance of the motor dysfunction experienced by males with ADHD-HI becomes apparent in a comparison of the categorical distribution according to their MABC centile ranking in Table IV. This result clearly demonstrated problematic motor dysfunction for the ADHID-HI males, with $31.3 \%$ found within the DCD level compared with $10.3 \%$ in the comparison group. It is not until the continuum symptom scores for inattentive symptomatology are examined (see 'lable l) that it becomes obvious that, despite not reaching the clinical threshold for diagnosis of inattention, this group still had higher levels of inattention than the comparison group. The strength of this finding is that it replicates the findings of Piek et al. (1999) and supports previous reports of the link between motor ability and inattention (c.g. Lyytinen and Ahonen 1989) highlighting the importance of this particular ADHD symptomatology as

Table VI: Means, SDs, and range for groups on components of Purdue Pegboard test (PPB)

\begin{tabular}{|c|c|c|c|c|}
\hline Tests & $\begin{array}{r}\text { Comparison } \\
(n=39)\end{array}$ & $\begin{array}{r}A D H D-P I \\
(n=50)\end{array}$ & $\begin{array}{r}A D H D-H I \\
(n=16)\end{array}$ & $\begin{array}{r}A D H D-C \\
(n=38)\end{array}$ \\
\hline \multicolumn{5}{|c|}{ PPB Dominant Hand ${ }^{a}$} \\
\hline Mean & 13.63 & 12.62 & 12.96 & 12.96 \\
\hline SD & 1.39 & 1.71 & 2.48 & 2.21 \\
\hline Range & $10-17$ & $9-17$ & $9-17$ & $8-19$ \\
\hline \multicolumn{5}{|c|}{ PPB Non-dominant Hand ${ }^{\mathrm{a}}$} \\
\hline Mean & 12.94 & 11.72 & 12.21 & 12.23 \\
\hline SD & 1.61 & 2.00 & 2.23 & 1.94 \\
\hline Range & $10-16$ & $7-16$ & $9-17$ & $9-19$ \\
\hline \multicolumn{5}{|c|}{ PPB Both Hands Together ${ }^{a}$} \\
\hline Mean & 10.70 & 9.79 & 9.63 & 10.11 \\
\hline $\mathrm{SD}$ & 1.48 & 1.61 & 2.45 & 1.81 \\
\hline Range & $8-14$ & $6-13$ & $6-14$ & $5-15$ \\
\hline \multicolumn{5}{|c|}{ PPB Composite ${ }^{a, b}$} \\
\hline Mean & 37.25 & 33.99 & 34.73 & 35.25 \\
\hline SD & 3.96 & 4.91 & 6.75 & 5.67 \\
\hline Range & $29-44$ & $22-46$ & $25-48$ & $23-53$ \\
\hline \multicolumn{5}{|c|}{ PPB Assembly ${ }^{\mathrm{a}}$} \\
\hline Mean & 27.92 & 24.87 & 25.79 & 25.46 \\
\hline SD & 5.74 & 5.40 & 9.60 & 6.74 \\
\hline Range & $15-41$ & $14-35$ & $8-40$ & $13-48$ \\
\hline
\end{tabular}

aHigher the score, better the performance; ${ }^{\mathrm{b}}$ sum of PPB Dominant, Non-dominant, and Both Hands scores. a determinant of the overall level of motor functioning.

The results of the current study, therefore, provide new evidence of significant motor impairment in all three DSM-IV ADHD subtypes. Around 50\% of children with each subtype of ADHD have motor performance problems, compared with $20 \%$ in the comparison group. (It should be pointed out that this proportion is quite large for the comparison group, and may be a function of the sampling as parents may have enrolled their children in the study if they were concerned about their child's motor ability.) In summary, whilst previous literature has pointed to a link between $\mathrm{ADHD}$ and $\mathrm{DCD}$, in particular for the ADHD-PI and ADHD-C subtypes (e.g. Hartsough and Lambert 1985; Piek et al. 1999), the current research has also identified that the motor problems are a shared feature of all DSM-IV subtypes and extend to the ADHD-HI group.

Each of the three subtests of the MABC was examined separately to determine any distinct performance profiles for the ADHD subtypes. The outcome for static and dynamic balance indicated that the groups did not significantly differ on this measure. This does not support the findings of Piek et al. (1999) who found a significant deficit in gross motor ability for the ADHD-C group as compared with the comparison group. This result is somewhat unexpected given the range of literature that has reported deficient gross motor ability as determined by neurological soft-sign tests (e.g. Carte et al. 1996, Barkley 1998, Nigg et al. 1998). Additionally, given that the presence of 'soft' neurological signs has been shown to improve or resolve with the use of methylphenidate (Lerer and Lerer 1976) and that the ADHD children within the current study were tested unmedicated, it seemed more likely that these differences would have been observed in the current study as opposed to the Piek et al. study (1999) in which over

Table VII: Means, SD, and range for groups on components of Purdue Pegboard test (PPB)

\begin{tabular}{lrrr}
\hline Tests & $\begin{array}{r}\text { Comparison } \\
(n=31)\end{array}$ & $\begin{array}{r}\text { ADHD } \\
(n=49)\end{array}$ & $\begin{array}{r}\text { ADHD/DCD } \\
(n=55)\end{array}$ \\
\hline PPB Dominant Hand & & & \\
Mean & & & 12.12 \\
SD & 13.78 & 13.56 & 1.91 \\
Range & 1.45 & 1.88 & $8-16$ \\
PPB Non-dominant Hand ${ }^{\mathrm{a}}$ & $10-17$ & $10-19$ & \\
Mean & 13.10 & 12.78 & 11.27 \\
SD & 1.66 & 1.96 & 1.78 \\
Range & $10-16$ & $9-19$ & $7-15$ \\
PPB Both Hands Together ${ }^{\mathrm{a}}$ & & & \\
Mean & 10.82 & 10.55 & 9.28 \\
SD & 1.54 & 1.71 & 1.73 \\
Range & $8-14$ & $8-15$ & $5-13$ \\
PPB Composite ${ }^{\mathrm{a}, \mathrm{b}}$ & & & \\
Mean & 37.66 & 36.88 & 32.50 \\
SD & 4.13 & 5.09 & 4.99 \\
Range & $29-44$ & $28-53$ & $22-43$ \\
PPB Assembly & & & \\
Mean & & 27.14 & 23.53 \\
SD & 28.56 & 6.42 & 6.37 \\
Range & 5.47 & $17-48$ & $8-40$ \\
\hline
\end{tabular}

${ }^{\mathrm{a}}$ Higher the score, better the performance; ${ }^{\mathrm{b}}$ sum of PPB Dominant, Non-dominant, and Both Hands scores. 
$60 \%$ of the ADHD-C children were medicated. However, in general, the use of soft neurological signs has received some criticism due to the high variability in outcomes for children with hyperactivity (McMahon and Greenburg 1977). The result with respect to gross motor dysfunction remains inconclusive.

The outcome for ball skills supported the prediction of a greater performance deficit for the ADHD-PI and ADHD-C group as compared with the comparison group. 'The ball skills of the ADHD-PI group were also, as predicted, significantly worse than those of the ADHI-HI group, yet the performance of the ADHD-C group was not found to differ significantly to that of either the ADHD-HI group or the ADHD-PI group. Also, the ADHD-HI group did not have significantly poorer ball skills than the comparison group. Despite previously referring to ball skills as a test of fine and gross motor skill, elements of visual processing may also relate to the performance on this specific motor task. Thus interpretation of the results may tend to imply that the visual motor skills of the groups significantly varied. The finding is in contrast to that of Carlson et al. (1986) who failed to find any significant visual-motor skill differences between children with attention-deficit disorder (ADD) with hyperactivity, ADD without hyperactivity, or their control groups. Additionally, the current finding does not support the findings of Frank and Ben-Nun (1988) that children with ADD with hyperactivity have poorer ball handling than children with ADD without hyperactivity. 'The IDSM-IV equivalent groups within the current study were not significantly different in their ball handling ability and inattentive symptomatology was again linked to poorer motor performance.

An examination of the manual dexterity scores demonstrated that the groups significantly differed according to their fine motor skills as measured by the MABC. Planned comparisons identified that, as predicted, the ADHD-PI group had significantly worse manual dexterity skills than the comparison group but not significantly different to the ADHD-C or ADHD-HI groups. As predicted, the ADHD-C group was also found to have significantly poorer fine motor skills than the comparison group but no worse than the ADHD-HI group. The ADHD-HI group also did not differ from the comparison group, although scores were suggestive of higher levels of dysfunction than the comparison group.

These results support, in part, the findings of Piek et al. (1999) in that, once again, a fine motor skill deficit was observed for the ADHD-PI group. However, in contrast to that study, the ADHD-C group experienced similar dysfunction. 'The results for the ADHD-C group in the Piek et al. study may well have been influenced by their positive test medicated status at the time of testing (i.e. stimulants may improve fine motor coordination; Shaywitz and Shaywitz 1984). Over $60 \%$ of their ADHD-C group, as compared with less than $20 \%$ of the ADHD-PI group, were medicated in the Piek et al. study and, consequently, the level of dysfunction in the ADHD-C group may have been dampened.

Fine motor ability was further investigated using the PPB. As predicted, the ADHD-PI group had significantly poorer fine motor skills on this measure than clid the comparison group. However, the prediction of poorer fine motor skills on this measure when compared with the ADHD-HI group was not supported. Furthermore, it was expected that the score for the ADHD-C group would be significantly worse than the mean score for either the ADHD-HI or comparison groups. However, the only significant finding for the PPB variables was the outcome for ADHD-PI on the PPB composite score. 'This result must be accepted with a degree of caution given that it occurred with a composite variable elevating the risk for 'Iype I error. However, the result is in line with expectation and supports the earlier findings for deficient fine motor skill as measured by the MABC manual dexterity task.

A further aim of the current study was to determine the impact of having both AIDHD and DCD, in contrast with AIDHD only, on fine motor performance as measured by the PPI3. Children with the categorization of both ADHD and DCD had consistently poorer performance than both the comparison group and the children with only ADHD on all of the fine motor tests of the PPB, even after equating groups on VIQ. All contrasts between the comparison group and the ADHD group were nonsignificant indicating that these groups did not differ in their fine motor ability. This finding demonstrates that poor fine motor ability is not a result of the ADHD symptomatology, but rather of the comorbid DCI condition. That is, the poor performance on the fine motor measures cannot be attributed to attention and concentration deficits, as has been argued in the past (e.g. Doyle et al. 1995), particularly when considering that there was no significant difference between the single and dual diagnosis groups in terms of their inattentive symptomatology (see Table II). These general findings for greater fine motor dysfunction for the males within the ADHD/DCD group support the use of the PPB test as a measure able to discriminate between the performance of AIHD groups with motor difficulties (i.e. DCD) and those without (i.e. no DCD). It also explains the confusing and often conflicting results found on motor measures when investigating ADHD without taking into account children who may also have DCD.

The findings of the current study point to a reciprocal need for not only the assessment of motor function within males with ADHD (Piek et al. 1999), but for the assessment of ADHI) symptomatology within children with DCD. The failure of the DSM-IV to recognize adequately motor difficulties within the ADHD section of the diagnostic manual suggests that up to

Table VIII: Statistical significance, effect size, and power for univariate ANCOVAs examining components of Purdue Pegboard (PPB; Tiffin and Asher 1948, Tiffin 1968) test with verbal IQ as a covariate

\begin{tabular}{lrrrrr}
\hline Test & $F$ & $d f$ & $p$ & Partial $\mu^{2}$ & Power \\
\hline PPB Dominant Hand & 8.99 & 2,131 & 0.000 & 0.121 & 0.971 \\
PPB Non-dominant Hand & 10.73 & 2,131 & 0.000 & 0.141 & 0.989 \\
PPB Both Hands Together & 8.27 & 2,131 & 0.000 & 0.112 & 0.958 \\
PPB Assembly & 4.88 & 2,131 & 0.009 & 0.069 & 0.796 \\
\hline
\end{tabular}


$50 \%$ of children with ADHD may also be experiencing all the secondary difficulties attributable to DCD without appropriate recognition or intervention. 'The potential exists for social, emotional, and behavioural difficulties associated with the movement dysfunction (e.g. negative appraisal or social derision; Henderson and Hall 1982, Cratty 1994, Schoemaker and Kalverboer 1994), physical weakness and low fitness (Cratty 1994, Bouffard et al. 1996), or other educational, social and emotional problems (Losse et al. 1991, Geuze and Börger 1993, Hellgren et al. 1993, Cantell et al. 1994, Skinner and Pick 2001) to be misattributed to the ADHD symptomatology (Vacssen and van der Meere 1990), although it is more likely that the combined effect of both conditions warrants close attention. A strong recommendation based on the current research is for the recognition of motor skills disorders such as DCD within the differential diagnosis section for ADHD. To continue to emphasize the attribution of any observed motor difficulty for children with ADHD to the symptoms of distractibility or impulsivity advocates the minimization of observed motor problems and their associated impact.

\section{DOI: 10.1017/\$0012162203000975}

\section{Accepted forpublication 23rd April 2003.}

\section{Acknowledgements}

This rescarch was principally supported by a scholarship from The Women's Service Guild (WSG) of Western Australia Trust and a private research grant provided by Mr lan and Mrs Jean IIutcheson to the first author. We wish to thank the participants, their parents, and the participating schools for their support of this research.

\section{References}

American Psychiatric Association. (1994) Diagnostic and Statistical Manual of Mental Disorders. 4th edn. Washington, DC: Amcrican Psychiatric Association.

American Psychiatric Association. (1987) Diagnostic and Statistical Manual of Mental Disorders. 3rd edn. Revised. Washington, DC: American Psychiatric Association

August (i.), Ostrander R, Bloomquist M.. (1992) Attention deficit hyperactivity disorder: an epidemiological screening method. AmJ (orthopsychiatry 62: 387-396.

Barkley RA. (1997) Bchavioural inhibition, sustained attention, executive functions - constructing a unifying theory of $\Lambda \mathrm{DHD}$. Psychol Bull 121: 65-94́

Barkley RA. (1998) Attention Deficit Hyperactivity Disorder: A Handbook for Diagnosis and 'Treatment. 2 nd edn. New York: Guilford Press.

Barkley RA, DuPaul GJ, McMurray MB. (1990) Comprehensive evaluation of attention deficit disorder with and without hyperactivity as defined by research criteria. J Consult Clin Psychol 58: 775-789

Bax M, Whitmore K. (1987) the medical examination of children on entry to school. The results and use of neurodevelopmental assessment. Dev Med Child Neurol 29: 40-55.

Bouffard M, Watkinson EJ, Thompson LP, Dunn JLC, Romanow SKR. (1996) A test of the activity deficit hypothesis with children with movement difficulties. Adapt Pbys Activ $Q$ 13: 61-73.

Cantell MH. (1998) Developmental coordination disorder in adolesence: perceptual-motor, academic and social outcomes of carly motor delay. Res Rep Sports Health 112 . Jyväskylä, Finland: LIKES - Research Center for Sport and Health Science.

Cantell MH, Smyth MH, Ahonen TP. (1994) Clumsiness in adolescence: cducational, motor, and social outcomes of motor delay detected at 5 years. Adapt Phys Activ $Q$ 11: 115-129.

Carlson CI. (1986) Attention deficit disorder without hyperactivity a review of preliminary experimental evidence. In: Lahey BB, Kardin AE, cditors. Advances in Clinical Cbild Psychology Vol. 9 New York: PIenum Press. p 153-175.
Carte ET, NiggJ'I, Hinshaw SP. (1996) Neuropsychological functioning, motor speed, and language processing in males with and without ADHD. J Abnorm Child Psychol 24: 481-499.

Conners CK. (1997) Conners' Rating Scales - Revised: Technical Manual. North Tonawanda, NY: Multi-Health Systems.

Conners CK, Sitarenios G, Parker JDA, Epstein JN. (1998) The revised Conners' Parent Rating Scale. J Abnorm Child Psychol 26: $257-268$.

Cratty BJ. (1994) Clumsy Child Syndromes: Descriptions, Evaluation and Remediation. Langhorne, PA: Harwood Academic.

Doyle S, Wallen M, Whitmont S. (1995) Motor skills in Australian children with attention deficit hyperactivity disorder. Occup Ther Int 2: 229-240.

Frank Y, Ben-Nun Y. (1988) Toward a clinical subgrouping of hyperactive and nonhyperactive attention deficit disorder.J Dis Child 142: 153-155.

Garcia C. (1994) Gender differences in young children's interactions when learning fundamental motor skills. Res $Q$ kxerc Sport 65: 213-246.

Gardner RA, Broman M. (1979) The Purdue Pegboard: normative data on 1334 school children./ Clin Psychol 1: 156-162.

Geuze, R, Börger H. (1993) Children who are clumsy: five years later. Adapt Phys Activ Q 10: 10-21.

Gillberg C. (1992) Deficits in attention, motor control and perception, and other syndromes attributed to minimal brain dysfunction. In: Aicardi J, editor. Diseases of the Nervous System in Children.Clinics in Developmental Medicine: No. 115-118. London: Mac Keith Press. p 1321-1337.

Gillberg C. (1995) Deficits in attention, motor control and perception, and other syndromes attributed to minimal brain dysfunction. In: Gillberg C, editor. Clinical Child Neuropsychiatry. New York, NY: Cambridge University Press. p $138-172$

Gillberg C. (1998) Hyperactivity, inattention and motor control problems: prevalence, comorbidity and background factors. Folia Phoniatr Logop 50: 107-117.

Gillberg C, Hellgren L. (1996) Outcome. In: S. Sandberg, editor Monographs in Child and Adolescent Psychiatry: Hyperactivity Disorders of Childbood. Cambridge, UK: Cambridge University Press. p 477-503.

I lart FI, Lahey BB, Loeber R, Applegate B, lrick PJ. (1995) Developmental changes in attention-deficit hyperactivity disorder in males: A four-year longitudinal study.J Abnorm Child Psychol 23: 729-750.

Hartsough CS, Lambert NM. (1985) Medical factors in hyperactive and normal children: prenatal, development, and health history findings. AmJ Ortbopsycbiatry 55: 190-210.

Hellgren I, Gillberg C, Gillberg IC, Enerskog I. (1993) Motor control and perception (DAMP) almost grown up: general health at 16 years. Dev Med Child Neurol 35: 881-892.

Hellgren L, Gillberg IC, Bagenholm $\Lambda$, Gillberg C. (1994) Children with deficits in attention, motor control and perception (DAMP) almost grown up - psychiatric and personality disorders at age 16 years. J Child Psychol Psycbiatry 35: 1255-1271.

Henderson SE, Hall D. (1982) Concomitants of clumsiness in young schoolchildren. Dev Med Ghild Neurol 24: 448-460.

Henderson SE, Sugden DA. (1992) Movement Assessment Battery for Children. New York: Psychological Corporation/Harcourt Brace-Jovanovich.

Hoare D, Larkin D. (1991) Coordination problems in children. National Sports Research Centre. No. 18. p 1-16.

Houghton S, Wheldall K, Jukes R, Sharpe A. (1990) The effects of limited private reprimands and increased private praise on classroom behaviour in four British secondary school classes. BritJEduc Psychol 60: 255-265.

Issac AR, Marks DF. (1994) Individual differences in mental imagery experience: developmental changes and specialisation. BritJ Psychol 84: 479-501.

Kadesjö B, Gillberg C. (1998) Attention deficit and clumsiness in Swedish 7-ycar-olds. Dev Med Child Neurol 40: 796-804.

lahey BB, Applegate B, McBurnett K, Biederman J, Greenhill L, Hynd GW, Barklcy RA, Newcorn J, Jensen P, Richters J, Garfinkel B, Kerdyk L, Frick PJ, Ollendick T, Pere\% D, Hart EL, Waldman I, Shaffer D. (1994) DSM-IV field trials for attention deficit hyperactivity disorder in children and adolescents. $A m J$ Psychiatr 151: 1673-1685. 
Lahey BB, Pelham WE, Stein MA, Loncy J, Trapani C, Nugent K, Kipp H, Schmidt E, Lee S, Cale M, Gold E, Hartung CM, Willcutt E, Baumann B. (1998) Validity of DSM-IV attention-deficit/hyperactivity disorder for younger children.J Am Acad Cbild Adolesc Psychiatr 3\%: 695-702

Laszlo JI, Bairstow PJ, Ward GR, Bancroft H. (1980) Distracting information, motor performance and sex differences. Nature 283: $37-38$

Lerer RJ, Lerer MP. (1976) The effects of methylphenidate on the soft neurological signs of hyperactive children. Pediatrics 5\%: $521-525$.

Levy F, Hay DA, editors. (2001) Attention Genes and ADHD. Philadelphia: Brunner-Routledge

L.evy F, Hay D, McLaughlin M, Wood C. Waldman I. (1996) The Australian If in Bebaviour Rating Scale. Unpublished rating scale.

Levy F, Hay D, McStephen M, Wood C, Waldman I. (1997) Attentiondeficit hyperactivity disorder: a category or a continuum? Genetic analysis of a large-scale twin study. J Am Acad Child Adolesc Psychiatr 36: 1-8

Levy F, McStephen M, Hay DA. (2001) Diagnostic genetics of ADH ID symptoms and subtypes. In F L.evy, DA Hay, editors. Attention, Genes and ADHDD Sydncy, NSW: Macmillan. p 35-57.

livesey DJ, Intili D. (1996) A gender difference in visual-spatial ability in 1-year-old children: effects of performance of a kinacsthetic acuity task. J Exp Child Psychol 63: 436-447.

Lord R, Hulme C. (1987) Kinaesthetic sensitivity of normal and clumsy children. Dev Med Child Neurol 29: 720-725

Losse A, Henderson SE, Flliman D, Hall D, Knight E, Jongmans, M. (1991) Clumsiness in children - do they grow out of it? A 10 year follow-up study. Dev Med Child Nezrol 33: 55-68.

Iyytinen $\mathrm{H}$, Ahonen T. (1989) Motor precursors of learning disabilities. In DJ Baker, II. van der Vlugo, editors. Learning Disabilities: Neuropsychological Correlates and Treatment (Vol. I) Amsterdam: Swets. p 135-143

McMahon SA Greenburg I.M. (1977) Serial neurological examination of hyperactive children. Pediatrics 59: 584-587.

Merett F, Wheldall K. (1986) Observing pupils and teachers in classrooms (OP'IC): a behavioural observation schedule for use in schools. Educ Psychol 6: 57-70.

Nigg J'I', I linshaw Sl, Carte, 'Ireuting JJ. (1998) Neuropsychological correlates of childhood attention-deficit/hyperactivity disorder: explainable by comorbid distuptive behavior or reading problems? J Abnor Psychol 10\%: 468-480

Pelham WE, Gnagy FM, Greenslade KE, Milich R. (1992) 'leacher ratings of DSM-III-R symptoms for the disruptive behavior disorders. J Am Acad Cbild Adolesc Psychialr 31: 210-218.

Pick JP, Colcman-Carman R. (1995) Kinaesthetic sensitivity and motor performance of children with developmental co-ordination disorder. Dev Med Cbild Neurol 3\%: 976-984.

Pick JP, Dworcan M, Barrett NC, Coleman R. (2000) Determinants of self-worth in children with and without developmental coordination disorder. Int J Disabil Dev Educ 4\%: 259-272.

Piek JP, Pitcher TM, Hay I)A. (1999) Motor coordination and kinaesthesis in males with attention-deficit-hyperactivity disorder. Dev Med Child Neurol 41: 159-165.

Polatajko HJ, Fox AM, Missiuna C. (1995) An international consensus on childeen with developmental coordination disorder. Canfoccup Ther 62: 3-6.

Rasmussen E, Neuman R, Heath A, levy F, Hay D, Todd R. (2002) Replication of the latent class structure of attentiondeficit/hyperactivity disorder (ADHD) subtypes in a sample of Australian twins. J Child Psychol Psychiatr 4: 1018-1028.

Rasmussen P, Gillberg C. (2000) Natural outcome of AIOYID with developmental coordination disorder at age 22 years: al controlled, longitudinal, community-based study. J Am Acad Child Adolesc Psycbiatr 39: 1424-1431.
Rutter M. (1982) Syndromes attributed to 'minimal brain dysfunction' in childhood. Am J Psychiatr 139: 21-33.

Sandberg SE. (1996) Monographs in Cbild and Adolescent Psychiatry: Hyperactivity Disorders of Cbildbood. Cambridge, UK: Cambridge University Press.

Sattler JM. (1992) Assessment of Children. 3rd edn. San Diego, CA JM Sattler.

Schoemaker MM, Kalverboer AF. (1994) Social and affective problems of children who are dumsy: how early do they begin? Adapt Pbys Activ $Q$ 11: 130-140.

Shaywitz SE, Shaywitz BA. (1984) Diagnosis and management of attention deficit disorder: a pediatric perspective. Pediatr Clin North Am 31: 429-457.

Skinner RA, PickJP. (2001) Psychosocial implications of poor motor coordination in children and adolescents. Hum Mov Sci 20: $73-94$.

Smits-Engelsman BCM, van Galen GP, Schoemakex MM. (1998) Theory-based diagnosis and subclassification in the developmental coordination disorder. In J. Rispens and $\mathrm{TAV}$ Yperen, W Yule, editors Perspectives on the Classification of Specific Developmental Disorders Dordrecht, Netherlands: Kluwer Academic. p 245-264.

Smyth 'T'R. (1992) Impaired motor skill (clumsiness) in otherwisc normal children: a review. Child: Care Health I)ev 18: 283-300,

Stott DII, Moyes IA, IIcnderson SI: (1984) The Fest of Molor Impairment. San Antonio, IX: 'l'he Psychological Corporation.

Iiffin J. (1968) Purdue Pegboard: Examiner Manual Chicago: Science Research Associates.

'Tiffin J, Asher EJ. (1948) The Purdue Pegboard: norms and studies of reliability and validity. $J$ Apl $/ P s y c h o l 32: 234-247$.

Vaessen W, van der Meere J. (1990) Issues in the selection of hyperactive/ADD children for experimental clinical studies. In $\Lambda \mathrm{F}$ Kalverboer, editor. Developmental Biopsychology: Lxperimenlal and Observational Studies in Children at Risk. Ann Arbor, MI University of Michigan Press. p 21-37.

Wechsler D. (1992) Manual for the Wechsler Intelligence Scale for Cbildren. 3rd edn. New York: Psychological Corporation

List of abbreviations

\begin{tabular}{ll}
\hline ADBS & Australian Disruptive Behaviours Scale \\
ADD & Attention-deficit disorder \\
ADD/H & Attention-deficit disorder with hyperactivity \\
ADHD-C & Attention-deficit-hyperactivity disorder, combined \\
& type \\
ADHD-HI & Attention-deficit-hyperactivity disorder, \\
& hyperactive/impulsive \\
ADHD-PI & Attention-deficit-hyperactivity disorder \\
& predominantly inattentive \\
CPRS-R:L & Conners Parent Rating Scale-Revised: Long form \\
DAMP & Deficits in attention, motor control, and perception \\
DCD & Developmental coordination disorder \\
MABC & Movement Assessment Battery for Children \\
PPB & Purdue Pegboard \\
TOMI & Test of Motor Impairment
\end{tabular}

